\title{
Effect of Shoe Inserts on Kinematics, Center of Pressure, and Leg Joint Moments during Running
}

\author{
BENNO M. NIGG, PRO STERGIOU, GERALD COLE, DARREN STEFANYSHYN, ANNE MÜNDERMANN, and \\ NEIL HUMBLE
}

Human Performance Laboratory, Faculty of Kinesiology, University of Calgary, Calgary, Alberta, CANADA

\begin{abstract}
NIGG, B. M., P. STERGIOU, G. COLE, D. STEFANYSHYN, A. MÜNDERMANN, and N. HUMBLE. Effect of Shoe Inserts on Kinematics, Center of Pressure, and Leg Joint Moments during Running. Med. Sci. Sports Exerc., Vol. 35, No. 2, pp. 314-319, 2003. Purpose: The purposes of this project were to assess the effect of four different shoe inserts on the path of the center of pressure (COP), to quantify the effect of these inserts on selected knee joint moments during running, and to assess the potential of COP data to predict the effects of inserts/orthotics on knee joint moments. Methods: Kinematics for the lower extremities, resultant ankle and knee joint moments, and the path of the COP were collected from the right foot of 15 male subjects while running heel-toe with five different shoe inserts (full or half with 4.5 -mm postings). Results: Individual movement changes with respect to the neutral insert condition were typically small and not systematic. Significant changes for the path of the COP were registered only for the full lateral insert condition with an average shift toward the lateral side. The mediolateral shift of the COP was not consistent for the full medial and the two half-shoe inserts. The subject-specific reactions to the inserts' intervention in the corresponding knee joint moments were typically not consistent. Compared with the neutral insert condition, subjects showed increases or decreases of the knee joint moments. The correlation between the individual COP shifts and the resultant knee joint moment was generally small. Conclusion: The results of this study showed that subject-specific reactions to the tested inserts were often not as expected. Additionally, reactions were not consistent between the subjects. This result suggests that the prescription of inserts and/or orthotics is a difficult task and that methods must be developed to test and assess these effects. Such methods, however, are not currently available. Key Words: ORTHOTICS, JOINT LOADING, RUNNING INJURIES, CENTER OF PRESSURE, SHOE ORTHOTICS, PREVENTION
\end{abstract}

$\mathrm{S}$ hoe inserts and foot orthotics have been advocated and successfully used for many years for sports and other physically intensive activities $(4,9,30)$. Their administration or prescription has been and is done for many different reasons, including reducing the frequency of movement related injuries (10), aligning the skeleton properly $(11,16)$, providing improved cushioning (11), improving comfort (26), improving the sensory feedback $(25,27)$, reducing muscle activity (18), correcting the center of the path of the pressure (COP) (15), and/or reducing the knee joint moments (32).

This paper concentrates on the effects of shoe inserts on the alignment of the lower extremities, the correction of the path of the COP, and the reduction of ankle and knee joint moments. The alignment concept, the concept that shoe

\footnotetext{
Address for correspondence: Benno M. Nigg, Dr.sc.nat., Human Performance Laboratory, Faculty of Kinesiology, University of Calgary, 2500 University Drive, Calgary, Alberta, Canada, T2N 1N4; E-mail: nigg@ucalgary.ca.

Submitted for publication May 2002.

Accepted for publication September 2002.
}

0195-9131/03/3502-0314/\$3.00/0

MEDICINE \& SCIENCE IN SPORTS \& EXERCISE ${ }_{\circledast}$

Copyright (C) 2003 by the American College of Sports Medicine

DOI: $10.1249 / 01 . M S S .0000048828 .02268 .79$ inserts and orthotics minimize extreme joint amplitudes in the frontal plane, has successfully been used for the clinical population (e.g., 15). However, there is no concluding evidence that this alignment concept is equally successful for the healthy population (19).

Shoe inserts, orthotics, and braces are used to reduce the loading in the structures of interest. They often attempt to reduce the loading in and around the ankle and the knee joint. The variable most likely relevant for joint loading is the resultant joint moment (1). Ankle joint moments have, to the author's knowledge, not been associated with the development of ankle joint injuries. However, it has been speculated generally that high knee joint moments with respect to tibial ab-adduction and tibial rotation are associated with the development of knee injuries $(12,17,29)$. Specifically, it has been shown in a prospective and a retrospective study (32) that knee joint moments are associated with the development of patella-femoral pain syndrome. Thus, in cases of overloading or injuries, one may want to reduce these knee joint moments and the corresponding internal forces and stresses. Possible strategies include braces (7) and shoe orthotics or inserts $(3,6,8,13,18,21,23,31)$. Orthotic interventions are sometimes constructed and validated based on measurements of the COP between the foot and the shoe sole $(5,14)$. However, it is not known currently (a) how various shoe inserts/orthotics do affect ankle or knee joint moments, (b) how large these changes are, (c) whether these 


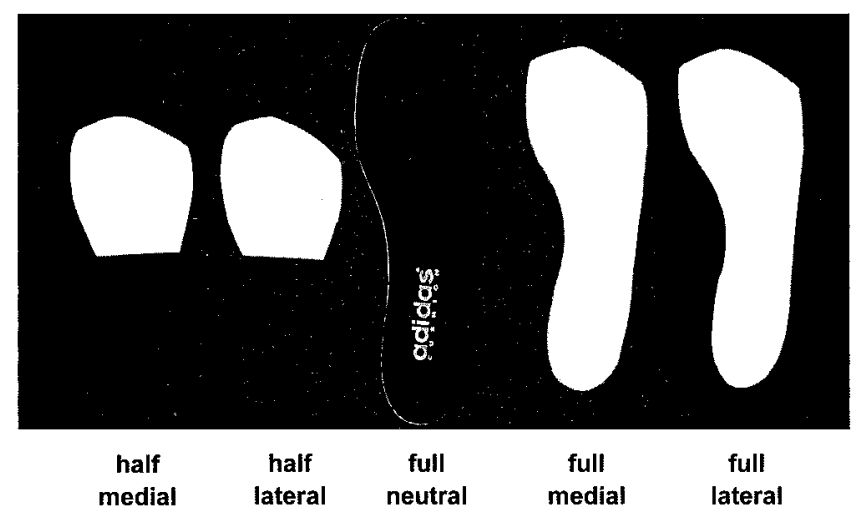

FIGURE 1-Illustration of the four posted and the neutral insert conditions used in the study.

effects are consistent for all subjects, and (d) whether the pressure distribution between the foot and the shoe can be used as an indicator for the effect of inserts/orthotics on knee joint moments.

Thus, the purposes of this project were to quantify the effect of four different shoe inserts (a) on lower extremity kinematics, (b) on the path of the COP, (c) on ankle and knee joint moments, and (d) to assess the potential of COP data to predict the effects of inserts/orthotics on knee joint moments for heel-toe running.

Specifically, the following hypotheses were tested. H1: The four shoe inserts produce systematic changes in the path of the COP during running. H2: The four shoe inserts produce systematic changes in ankle and knee joint moments during running. H3: The path of the COP as a result of shoe inserts can be used to predict changes in knee joint moments.

\section{METHODS}

Fifteen male subjects participated in the study. All subjects were free from recent lower-extremity injury or pain. Informed written consent in accordance with the University of Calgary's Ethics Committee was obtained from all subjects.

Data were collected on the right foot of each subject while running heel-toe with five different shoe inserts in a running shoe (Adidas Universal, size 9.5 or 10.5 U.S.). Four shoe inserts were prepared by a podiatrist using EVA material (shore C 80) and are called insert conditions in this text. All insert conditions had $4.5-\mathrm{mm}$ postings, either medially or laterally. The four insert conditions included full lateral, full medial, half lateral, and half medial (Fig. 1). One shoe insert consisted of the neutral insert that was delivered with the shoe. This condition is called the neutral condition in this text. The inserts were placed in the shoe underneath the sock-liners. The order of the insert and neutral conditions was randomly assigned and five running trials $(4.0 \pm 0.2$ $\mathrm{m} \cdot \mathrm{s}^{-1}$ ) were collected for each condition. The running speed of the subjects was monitored with photocells placed before and after the force plate.

Kinematics and kinetics. Ground reaction force data were collected with a Kistler force platform (Kistler AG,

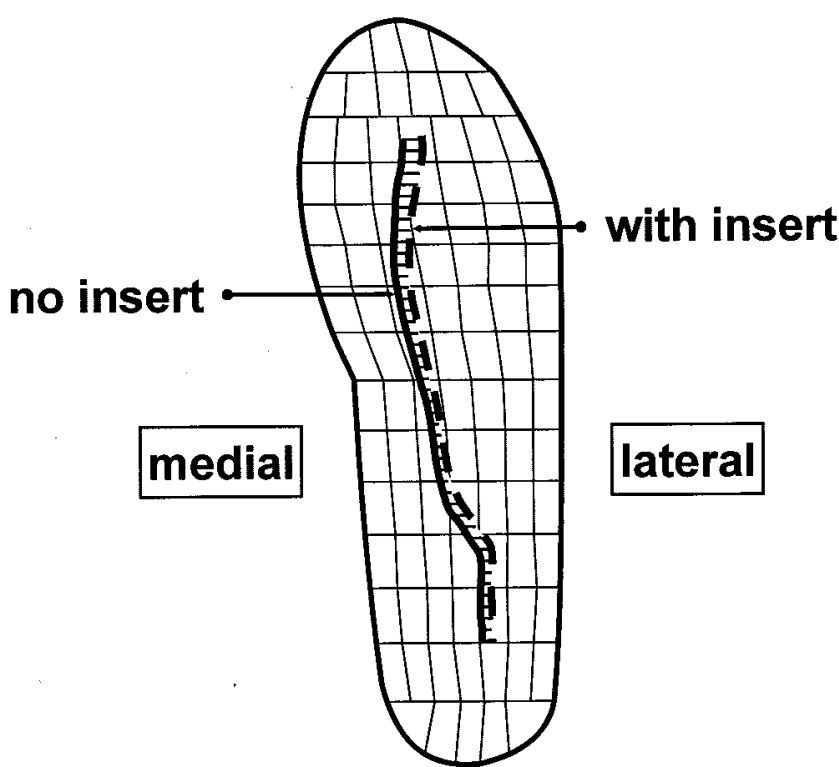

FIGURE 2-Illustration of the path of the COP determined from in-shoe pressure measurements for a neutral insert condition (solid line) and a selected insert condition (dashed line).

Winterthur, Switzerland) sampling at $1200 \mathrm{~Hz}$. Kinematic data were collected simultaneously with the kinetic data using a Motion Analysis six video camera system (Motion Analysis Corporation, Santa Rosa, CA) at $240 \mathrm{~Hz}$. Spherical reflective markers were placed on the thigh, shank, and shoe for kinematic data collection. All data were filtered using a low-pass fourth-order Butterworth filter. Cut-off frequencies were $12 \mathrm{~Hz}$ for the video data and $100 \mathrm{~Hz}$ for the force data. For the stance phase of running, data were normalized to 101 points.

COP. Pressure distribution data under the plantar surface of the right foot were collected with a PEDAR (Novel, Munich, Germany) pressure insole system with 99 sensors. Five trials were collected for each insert condition at a frequency of $99 \mathrm{~Hz}$. All COP data were normalized to 101 points corresponding to the stance phase of running. The variable used to describe the change in the COP path was the average mediolateral shift of the COP path, $\Delta \mathrm{x}_{\text {med-at }}$, with medial defined as positive (Fig. 2) for the initial, $\Delta \mathrm{x}_{\text {med- }}$ lat(init), and the stance phase, $\Delta x_{\text {med-lat }}($ mid). The initial phase was defined from $10 \%$ of ground contact to the time point when the COP path crossed an arbitrarily defined longitudinal shoe midline. The stance phase was defined from the end of the initial phase to $90 \%$ of ground contact.

Ankle and knee joint moments. A standing trial with additional markers before each set of running trials was used to define the joint centers. Additional markers were placed on the medial and lateral malleoli and the Achilles tendon to define the ankle joint center. Additional markers were placed on the lateral and medial epicondyles and the middle of the patella to define the knee joint center. A standard inverse dynamics approach was used to calculate resultant joint moments at the knee joint (Kintrak 6.0, The University of Calgary, Calgary, Canada). An average of all five trials collected was used for analysis. The variables used to de- 
scribe the knee joint moments were the maximal moment for knee abduction, $\mathrm{M}_{\mathrm{ab}}$, the maximal moment for external knee rotation, $\mathrm{M}_{\text {exrot}}$, and the changes of these moments due to the insert intervention (insert - neutral), $\Delta \mathrm{M}_{\mathrm{ab}}$ and $\Delta \mathrm{M}_{\text {exrot }}$.

Correlation between COP and knee joint moments. The correlation between the mean change in the path of the COP (from 10 to $90 \%$ of ground contact) and the mean change in the knee joint moments was determined for each individual subject. These correlations included five conditions per subject, with each condition representing one insert condition.

Statistics. Differences between insert conditions were determined using a paired $t$-test with a confidence level of $P<0.05$.

\section{RESULTS}

\section{Movement}

Significant changes for movement variables compared with the neutral condition were registered for several insert conditions.

Plantarflexion. The initial plantarflexion angle (angle between the sole of the shoe and the leg) was higher for the full medial $\left(+1.2 \pm 1.1^{\circ}\right)$ and the full lateral $\left(+1.0 \pm 1.4^{\circ}\right)$ insert condition. The maximal plantarflexion position was higher for the full lateral $\left(+0.7 \pm 1.3^{\circ}\right)$, the half medial $\left(+0.9 \pm 1.1^{\circ}\right)$ and the half lateral $\left(+1.1 \pm 1.5^{\circ}\right)$ insert condition. The total plantarflexion movement was lower for the full medial insert condition $\left(-0.9 \pm 1.3^{\circ}\right)$. However, these differences were small.

Shoe-leg in-eversion. The initial shoe-leg inversion position was higher for the full lateral insert condition $(+1.8$ $\left.\pm 2.1^{\circ}\right)$. The maximal shoe-leg eversion position was smaller for the full medial insert condition $\left(-1.5 \pm 1.3^{\circ}\right)$. The total shoe-leg eversion movement was smaller for the full medial $\left(-2.0 \pm 1.5^{\circ}\right)$ and higher for the full lateral $\left(+2.1 \pm 1.7^{\circ}\right)$ insert condition.

Individual movement changes with respect to the neutral insert condition were typically small and not systematic. The percentages of subject-insert combinations that showed changes that were higher than an arbitrarily selected limit of $3^{\circ}$ were $3.5 \%$ for initial dorsi-plantarflexion position, $3.5 \%$ for maximal dorsi-plantarflexion position, $12.3 \%$ for total plantarflexion movement, $14.0 \%$ for initial foot-leg inversion, $3.5 \%$ for maximal foot-leg eversion position, $15.8 \%$ for total foot-leg eversion movement, $26.3 \%$ for initial knee angle, none for maximal knee flexion, and $19.3 \%$ for total knee flexion movement. Thus, the largest effect was on the initial knee flexion and on the total knee flexion movement.

\section{COP}

Compared with the neutral insert condition, significant changes for the path of the COP were registered only for the full lateral insert condition. The initial mediolateral shift (mean and SD) of the COP, $\Delta \mathrm{x}_{\text {med-lat }}$ (init), was consistent for all subjects $(-2.3 \pm 1.8 \mathrm{~mm})$. The shift was toward the lateral side. For the same insert condition, the mediolateral

\section{$\Delta \mathbf{x}$ (initial)}

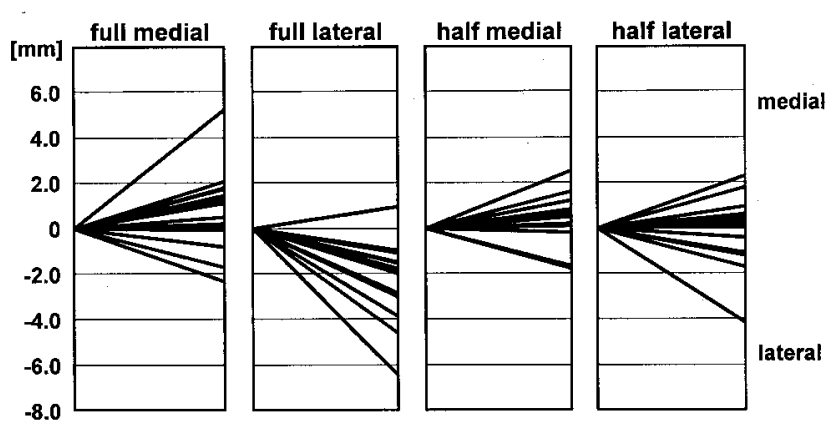

\section{$\Delta \mathbf{x}($ stance)}

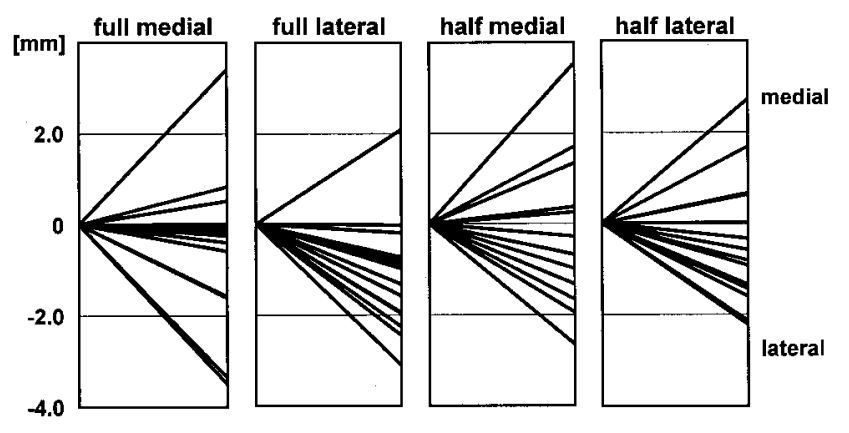

FIGURE 3-Mediolateral shift of the COP path due to the intervention with four different inserts, a full medial, a full lateral, a half medial, and a half lateral for the initial phase (top) and the midstance phase (bottom). The changes are with respect to the neutral insert condition. A positive result indicates a shift toward the medial side, a negative result a shift toward the lateral side.

shift of the COP during the midstance, $\Delta \mathrm{x}_{\text {med-lat }}(\mathrm{mid})$, was consistent for all but one subject $(-1.1 \pm 1.2 \mathrm{~mm})$. The average shift was toward the lateral side.

The mediolateral shift of the COP was not consistent for the full medial and the two half shoe inserts (Fig. 3). Some subjects showed a medial and some a lateral shift of the COP. Thus, hypothesis H1, stating that the four shoe inserts produce systematic changes in the path of the COP during running, was supported for the full lateral insert but must be rejected for the other three inserts.

\section{Moments}

Significant changes for ankle and knee joint moments compared with the neutral condition were registered for several insert conditions. Compared with the neutral insert condition, the maximal ankle joint inversion moments were significantly smaller for the full medial insert condition $(-3.4 \pm 5.9 \mathrm{~N} \cdot \mathrm{m})$. Compared with the neutral insert condition, the mean changes for all insert conditions (Fig. 4) were less than $2 \%$ for the knee extension moments and less than $7 \%$ for the knee abduction moments and not significant. The only significant group change due to inserts was measured for the maximal external knee rotation moment. Compared with the neutral insert condition, it was $27.6 \%$ 


\section{Knee joint moment}

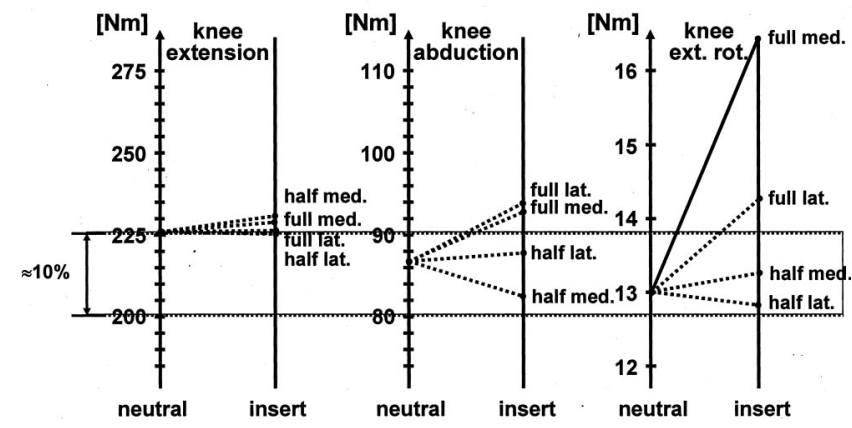

FIGURE 4-Mean group changes for the knee moments for the different insert conditions compared with the neutral insert condition. The scales for the three knee moment components have been chosen so that similar percentage changes correspond to each other. This has been illustrated with the $\approx 10 \%$ bars

higher for the full medial insert condition $(+3.6 \pm 3.2 \mathrm{~N} \cdot \mathrm{m})$ but less than $10 \%$ and not significantly different for the other insert conditions. Thus, hypothesis $\mathrm{H} 2$, stating that the four shoe inserts would produce systematic changes in ankle and knee joint moments during running could only be supported for (a) the ankle joint inversion moment for the full medial insert condition (corresponding to a decrease) and (b) for the external rotation knee joint moments for the full medial insert (corresponding to an increase) and could not be supported for the other insert interventions.

Individual moment changes with respect to the neutral insert condition were different for each rotational moment axis. The percentages of subject-insert combinations that showed changes that were higher than an arbitrarily selected limit of $10 \%$ were small for the maximal ankle plantarflexion moment $(7.0 \%)$ and the maximal knee extension moment $(12.3 \%)$. They were substantial for the maximal ankle inversion moment (57.9\%), the maximal ankle adduction moment (78.9\%), the maximal knee abduction moment $(47.4 \%)$, and the maximal external knee rotation moment (75.4\%).

However, the subject specific reactions to inserts in the knee joint moments were typically not consistent. Compared with the neutral insert condition, subjects showed increases or decreases of the knee joint moments. The corresponding numbers for the maximal knee abduction moment were 9/6 (9 increases and 6 decreases) for the full medial, $9 / 6$ for the full lateral, $5 / 6$ for the half medial, and $7 / 8$ for the half lateral insert condition. The corresponding numbers for the maximal external knee rotation moment were $13 / 2$ for the full medial, $9 / 6$ for the full lateral, $6 / 5$ for the half medial, and $6 / 9$ for the half lateral insert condition (Fig. 5).

\section{COP and Knee Joint Moments}

The correlation coefficients between the shift of the COP and the changes in knee joint moments were not consistent. Most subjects showed a low correlation between the COP shift (initial, stance, and total) and the change in moments, and only a few showed a high correlation (Table 1). Thus,

\section{Max. abduction moment (knee)}

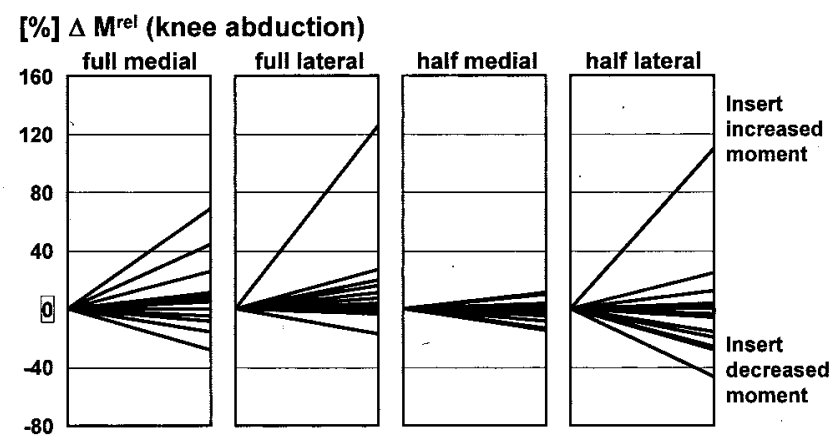

\section{Max. external rotation moment (knee)}

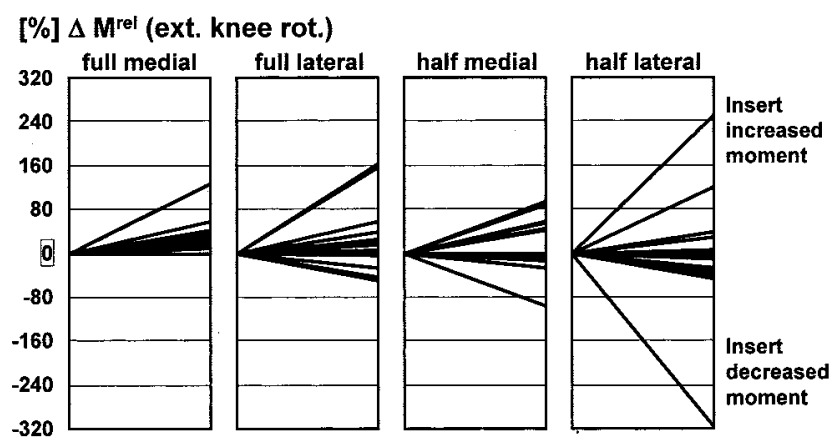

FIGURE 5-Illustration of the subject specific reactions to inserts for the maximal knee abduction moment (top) and the maximal external knee rotation moment (bottom).

hypothesis 3 , stating that the path of the COP as a result of shoe inserts can be used to predict changes in knee joint moments, could not be supported.

\section{DISCUSSION}

Thus, the purposes of this project were to quantify the effect of four different shoe inserts on lower-extremity kinematics, on the path of the COP, on ankle and knee joint moments, and to assess the potential of COP data to predict

TABLE 1. Correlation coefficients for the individual results for the shift of the COP and the resultant knee joint moments for abduction and external tibial rotation for all 15 test subjects.

\begin{tabular}{ccc}
\hline $\begin{array}{c}\text { Subject } \\
\text { No. }\end{array}$ & $\begin{array}{c}\text { Correlation } \\
\text { COP vs } \mathbf{M}_{\text {abduct }}\end{array}$ & $\begin{array}{c}\text { Correlation } \\
\text { COP vs } \mathbf{M}_{\text {extrot }}\end{array}$ \\
\hline 1 & 0.39 & 0.07 \\
2 & 0.68 & 0.19 \\
3 & 0.04 & 0.05 \\
4 & 0.02 & 0.34 \\
5 & 0.14 & 0.08 \\
6 & 0.25 & 0.50 \\
7 & 0.10 & 0.14 \\
8 & 0.52 & 0.52 \\
9 & 0.03 & 0.16 \\
10 & 0.85 & 0.90 \\
11 & 0.18 & 0.05 \\
12 & 0.12 & 0.02 \\
13 & 0.24 & 0.02 \\
14 & 0.00 & 0.03 \\
15 & 0.19 & 0.48 \\
\hline
\end{tabular}


the effects of inserts/orthotics on knee joint moments for heel-toe running. The findings of this study revealed some expected and many unexpected results.

The effects of the chosen insert interventions on the individual movement characteristics were small and not systematic. This result is in agreement with the results of many previous studies $(8,13,18,20-23,30)$. One may speculate that these small changes in kinematics due to the used shoe inserts is another support for the concept that the skeletal movement for a given movement task (e.g., heel-toe running) is preprogrammed (19) and that the goal of aligning the skeleton is not achieved through insert interventions in the normal healthy population.

Another surprising result was the reaction of the individual test subjects with respect to the path of the COP. We expected that the full lateral insert would shift the COP path to the medial side. However, the opposite was the case (Fig. 3). All but one subject reacted to the full lateral insert condition by shifting the COP path laterally. Thus, the intervention produced exactly the opposite result than expected. For the other three insert conditions, the reactions were randomly scattered. Thus, the results of this study suggest that insert strategies such as the tested medial or lateral postings cannot be used consistently to shift the COP path into a desired direction. In some cases, the subjects reacted as expected. However, in about $50 \%$ of all cases, the subjects reacted exactly opposite than expected.

The subject specific changes in the ankle and knee joint moments for the various insert interventions were not systematic. The relative changes were small for the foot plantarflexion and for the knee extension moments, the moments that are primarily responsible for the movement task of running. However, the effects were substantial for the ankle inversion and the ankle adduction moments, and for the knee abduction and the external knee rotation moments. The ankle inversion and adduction moments are much smaller than the ankle plantarflexion moments. The knee abduction and external rotation moments are much smaller than the knee extension moments. One may argue that the larger moments (ankle plantarflexion and knee extension) are primarily responsible for the actual locomotion, whereas the smaller moments are primarily responsible for the dynamic stability of the lower extremities. The result that was probably most surprising was that only one insert intervention, the full medial insert,

\section{REFERENCES}

1. Andriacchi, T. P. Dynamics of knee malalignment. Orthop. Clin. North Am. 25:395-403, 1994.

2. Barton, J. A. G., and A. Lees. Comparison of shoe insole materials by neural network analysis. Med. Biol. Eng. Comp. 34:453-459, 1996.

3. Bates, B. T., L. R. Osternig, B. Mason, and L. S. James. Foot orthotic devise to modify selected aspects of lower extremity mechanics. Am. J. Sports Med. 7:338-342, 1979.

4. Cavanagh, P. R. The Running Shoe Book. Mountain View, CA: Anderson World, Inc. 1980, pp. 1-385.

5. Chen, H., B. M. NigG, and J. J. DE Koning. Relationship between plantar pressure distribution under the foot and insole comfort. Biomech. 9:335-341, 1994. produced a reaction that was consistent and significant (Fig. 4). However, the full medial insert produced a significant increase (not decrease) in the external knee rotation moment. Thus, the conclusions from these results is (a) that the tested insert interventions did not affect the ankle and knee joint moments responsible for the actual locomotion, (b) that the selected interventions affected the ankle and knee joint moments more associated with the dynamic stability, and (c) that none of the tested insert intervention could be used consistently to reduce knee joint moments. Different subjects reacted differently to a specific insert intervention, and the reaction cannot, at this point in time, be predicted and the authors do not have a conclusive explanation for the results.

One of the goals of this study was to establish the relationship between the measured COP path and the moments for knee abduction and external knee rotation. Knee joint moments have been shown to be associated with the development of patellofemoral pain syndrome (32). However, the results of this study showed no correlation between changes in the path of the COP and changes in knee joint moments. Thus, isolated results from pressure distribution at the plantar surface of the shoe should not be used to predict changes in knee joint moments.

The reaction of subjects to insert interventions is influenced by many different factors, including mechanical $(3,9,14,31)$, neurophysiological $(2,5,24,28)$, anatomical, and maybe even psychological. Different subjects use different strategies, and it has been shown in this study conclusively that identical insert interventions produce substantially different results for different subjects. It is suggested that there are groups of subjects (functional groups) that produce similar reactions to specific insert interventions. Thus, it is of importance that future research concentrates on identifying these functional groups). Insert prescription is done frequently with success. However, predictions of the effects of specific insert interventions are currently not possible.

The results of this study also showed that subject specific reactions to the tested inserts were often not as expected. Additionally, reactions were not consistent between the subjects. This result suggests that the prescription of inserts and/or orthotics is a difficult task and that methods must be developed to test and assess these effects. Such methods, however, are not currently available.

6. Crenshaw, S. J., F. E. Pollo, and E. F. Carlton. Effects of lateral-wedged insoles on kinetics at the knee. Clin. Orthop. Relat. Res. 375:185-192, 2000

7. Devita, P., M. Torry, K. L. Glover, and D. L. Speroni. A functional knee brace alters joint torque and power patterns during walking and running. J. Biomech. 29:583-588, 1996.

8. Eng, J., and M. R. Pierrynowski. The effect of soft foot orthotics on three-dimensional lower-limb kinematics during walking and running. Phys. Ther. 74:836-844, 1994.

9. Gross, M. L., L. B. Davlin, and P. M. Evanski. Effectiveness of orthotic shoe inserts in the long-distance runner. Am. J. Sports Med. 19:409-412, 1991. 
10. Gross, M. L., R. C. NAPOLI. Treatment of lower extremity injuries with orthotic shoe inserts: an overview. Sports Med. 15:66-70, 1993.

11. Lockard, M. A. Foot orthoses. Phys. Ther. 68:1866-1873, 1988.

12. Mcclay, I., and K. Manal. Three-dimensional kinetic analysis of running: significance of secondary planes of motion. Med. Sci. Sports Exerc. 31:1629-1637, 1999.

13. Mcculloch, M., D. Brunt, and D. V. Linden. The effect of foot orthotics and gait velocity on lower limb kinematics and temporal events of stance. J. Orthop. Sport Phys. Ther. 17:2-10, 1993.

14. Mcnair, P. J., and R. N. Marshall. Kinematic and kinetic parameters associated with running in different shoes. Br. J. Sports Med. 28:256-260, 1994

15. Mcroil, T. G., M. Adrian, and P. Pidcoe. Effects of foot orthoses on center-of-pressure patterns in women. Phys. Ther. 69:149-154, 1989.

16. Milani, T. L., G. Schnabel, and E. M. Hennig. Rearfoot motion and pressure distribution pattern during running in shoes with varus and valgus wedges. J. Appl. Biomech. 11:177-187, 1995.

17. Morrey, B. F. Upper tibial osteotomy for secondary osteoarthritis of the knee. J. Bone Joint Surg. 71(B):554-559, 1989.

18. Nawoczenski, D. A., T. M. Cook, and C. L. Saltzman. The effect of foot orthotics on three-dimensional kinematics of the leg and rearfoot during running. J. Orthop. Sport Phys. Ther. 21:317-327, 1995.

19. NigG, B. M. The role of impact forces and foot pronation: a new paradigm. Clin. J. Sport Med. 11:2-9, 2001.

20. Nigg, B. M., H. A. Bahlsen, S. M. Luethi, and S. Stokes. The influence of running velocity and midsole hardness on external forces in heel-toe running. J. Biomech. 20:951-959, 1987.

21. Nigg, B. M., A. Kahn, V. Fisher, and D. Stefanyshyn. Effect of shoe insert construction on foot and leg movement. Med. Sci. Sports Exerc. 30:550-555, 1998.

22. Nigg, B. M., and M. Morlock. The influence of lateral heel flare of running shoes on pronation and impact forces. Med. Sci. Sports Exerc. 19:294-302, 1987.
23. Novick, A., and D. L. Kelley. Position and movement changes of the foot with orthotic intervention during the loading response of gait. J. Orthop. Sport Phys. Ther. 11:301-312, 1990.

24. Nurse, M. A., and B. M. NIGg. Quantifying a relationship between tactile and vibration sensitivity of the human foot with plantar pressure distribution during gait. Clin. Biomech. 14:667-672, 1999.

25. Nurse, M. A., and B. M. Nigg. The effect of changes in foot sensation on plantar pressure and muscle activity. Clin. Biomech. 16:719-727, 2001.

26. Pfeffinger, L. L. Foot orthoses. In: Atlas of Orthotics: Biomechanical Principles and Applications, American Academy of Orthopaedic Surgeons (Ed.). St. Louis: Mosby, 1975, pp. 346-357.

27. Robbins, S. E., and G. J. Gouw. Athletic footwear: unsafe due to perceptual illusions. Med. Sci. Sports Exerc. 23:217-224, 1991.

28. Robbins, S., E. Waked, P. Allard, J. Mcclaran, and N. Krouglicof. Foot position awareness in younger and older men: the influence of footwear sole properties. J. Am. Geriatr. Soc. 45:6166, 1997.

29. Scott, S. H., and D. A. Winter. Internal forces of chronic running injury sites. Med. Sci. Sports Exerc. 22:357-369, 1990.

30. Smith, L. S., T. E. Clarke, F. Santopietro, and C. L. Hamill. The effects of soft and semi-rigid orthotics upon rearfoot movement in running. J. Am. Podiatr. Med. Assoc. 76:227-233, 1986.

31. Stacoff, A., C. Reinschmidt, B. M. Nigg, et al. Effects of foot orthoses on skeletal motion during running. Clin. Biomech. 15: 54-64, 2000.

32. Stefanyshyn, D. J., P. Stergiou, V. M. Y. Lun, W. H. Meeuwisse, and B. M. NigG. Knee joint moments and patellofemoral pain syndrome in runners; part 1: a case control study; part 2: a prospective cohort study. Proc. 4th Symposium on Footwear Biomechanics, Canmore, Canada, 1999, pp. 86-87. 